\title{
THE CHOW COHOMOLOGY OF AFFINE TORIC VARIETIES
}

\author{
DAN EDIDIN AND RYAN RICHEY
}

\begin{abstract}
We study the Fulton-Macpherson Chow cohomology of affine toric varieties. In particular, we prove that the Chow cohomology vanishes in positive degree. We prove an analogous result for the operational $K$-theory defined by Anderson and Payne.
\end{abstract}

\section{INTRODUCTION}

Toric varieties are a rich source of examples for studying delicate questions in both ordinary and equivariant intersection theory. Both the Chow and cohomology rings of smooth, projective toric varieties are isomorphic to the Stanley-Reisner ring of the associated fan, while Fulton and Sturmfels [FS] proved that the Chow cohomology ring of a complete toric variety is the ring of Minkowski weights. Later, Payne [Pay] calculated the T-equivariant Chow cohomology ring of an arbitrary toric variety and identified it with the ring of piecewise polynomial functions on the corresponding fan. However, as noted by Katz and Payne $[\mathrm{KP}]$, the relationship between equivariant and ordinary Chow cohomology is quite subtle.

The main result of this paper, Theorem 4.1, is to show that the (non-equivariant) Chow cohomology ring of any affine toric variety vanishes in degree greater than zero. By contrast, the Chow homology groups of an affine toric variety need not vanish and can have both torsion and non-torsion elements.

Although their coordinate rings have prime cycles which are not rationally equivalent to 0 , our result shows that the Chow cohomology rings of affine toric varieties behave like that of affine spaces. As observed by Gubeladze Gub singular affine toric varieties constitute a class of intuitively contractible varieties naturally generalizing affine spaces. In particular, all vector bundles are trivial so the Grothendieck ring is isomorphic to $\mathbb{Z}$. In a parallel result we also prove, Theorem 5.1, that the operational $K$-theory ring defined by Anderson and Payne in $\mathrm{AP}$ is also isomorphic to $\mathbb{Z}$. Again, the Grothendieck group of coherent sheaves on an affine toric variety need not be isomorphic to $\mathbb{Z}$; see Example 5.7 .

Our Chow cohomology result fits into a framework developed in [ES]. There, the first author and Satriano show that for spaces, such as toric varieties, which are good moduli spaces of smooth Artin stacks, elements of the Chow cohomology ring have canonical representatives as topologically strong cycles on the corresponding stack.

Based on the results of [ES] the authors of the present paper have a conjectural description of the Chow cohomology ring of an arbitrary toric variety in terms of strong complete intersection cycles on the corresponding canonical stack. This will be presented elsewhere [Ric], but we remark that our results here imply the conjecture for affine toric varieties.

1.1. Outline of the proof. The proof of Theorem 4.1 uses Kimura's fundamental exact sequence for Chow cohomology [Kim and the stack-theoretic results proved in [ES].

2010 Mathematics Subject Classification. 14C15, 14F43, 14M25.

The first author was supported by Simons Collaboration Grant 315460 . 
If $X(\sigma)$ is the affine toric variety defined by a cone $\sigma$, then we denote by $\sigma^{*}$ the fan obtained by star subdivision with respect to the ray $v=\operatorname{Star}(\sigma)$. The map of toric varieties $X\left(\sigma^{*}\right) \rightarrow X(\sigma)$ is a blowup with exceptional divisor $E=V\left(\rho_{v}\right)$. Using Kimura's exact sequence for Chow cohomology, we show that in order to prove that $A_{\mathrm{op}}^{k}(X(\sigma))=0$, it suffices to prove the restriction map $A_{\mathrm{op}}^{k}\left(X\left(\sigma^{*}\right)\right) \rightarrow A_{\mathrm{op}}^{k}(E)$ is injective.

Denote by $\mathcal{E}$ and $\mathcal{X}\left(\sigma^{*}\right)$ the corresponding toric canonical stacks. Using the results of [ES] we can show that $A^{k}(E)_{\mathbb{Q}} \rightarrow A^{k}\left(X\left(\sigma^{*}\right)\right)_{\mathbb{Q}}$ is injective by showing that the corresponding map of Chow rings of smooth stacks $A^{k}\left(\mathcal{X}\left(\sigma^{*}\right)\right) \rightarrow A^{k}(\mathcal{E})$ is injective. By computing the Chow rings of toric canonical stacks we show directly that the corresponding map is in fact an isomorphism. This shows that $A^{k}(X(\sigma))_{\mathbb{Q}}=0$ for $k>0$. The proof concludes by using results of Hausel and Sturmfels to show that Chow cohomology of any affine toric variety is torsion free.

The proof for operational $K$-theory uses a similar stategy. Here we combine Anderson and Payne's $\mathrm{AP}$ results with Theorem 5.4 to obtain the desired result.

1.2. Acknowledgments. The authors are grateful to Sam Payne for suggesting that the corresponding result for operational $K$-theory should hold.

\section{BACKGROUND ON TORIC VARIETIES AND CANONICAL STACKS}

2.1. Basic toric terminology. Following [Ful2] or [CLS], let $k$ be be an algebraically closed field of characteristic zero, $N$ and $M$ be rank $n$ dual lattices over $\mathbb{Z}$ (which will be the lattices of 1-parameter subgroups and characters of a torus), and let $\sigma$ be a strongly convex, rational, and polyhedral cone in $N_{\mathbb{R}}=N \otimes_{\mathbb{Z}} \mathbb{R}$. From $\sigma$, we can construct the dual cone $\check{\sigma}$ in $M_{\mathbb{R}}$ which satisfies the property that $\check{\sigma} \cap M$ is a semigroup. The affine toric variety $X(\sigma)$ is defined as: $X(\sigma):=\operatorname{Spec}(k[(\sigma \check{\sigma} \cap)])$.

A fan $\Delta$ in $N_{\mathbb{R}}$ is a collection of cones satisfying the following two properties: each face of a cone is in $\Delta$, and the intersection of every two cones is a face of each. Each cone in $\Delta$ generates an affine toric variety which glue together to define a normal toric variety $X(\Delta)$ with an action of the torus $T_{N}=X(\{0\})$. Conversely, it is well known that every normal variety containing a dense torus whose action extends to an action on the total variety arises from a fan.

The orbit-cone correspondence associates to each cone $\sigma$ in a fan a $\Delta$ a torus orbit $O(\sigma)$ of dimension $n-\operatorname{dim} \sigma$. We denote the closure of $O(\sigma)$ by $V(\sigma)$. The orbit closure is a finite union of orbits corresponding to the faces of $\sigma$.

Torus equivariant morphisms of toric varieties $X\left(\Delta^{\prime}\right) \rightarrow X(\Delta)$ correspond to morphisms of lattices $\phi: N^{\prime} \rightarrow N$ which send cones of $\Delta^{\prime}$ into cones of $\Delta$. Various algebraic-geometric properties of the morphism can be understood in terms of conditions on the associated map of lattices. In particular, properness is equivalent to the condition that $\phi^{-1}(|\Delta|)=\left|\Delta^{\prime}\right|$. If $\phi$ is the identity map, then we say $\Delta^{\prime}$ is a refinement of $\Delta$. In what follows, we will be interested in the inverse image of a closed subscheme made up of orbit closures, especially in the case that $\Delta^{\prime}$ is a refinement of $\Delta$.

Theorem 2.1. [CLS, Lemma 3.3.21 and Theorem 11.1.10] With notation as above, suppose $\sigma$ is a cone in $\Delta$ with orbit closure $V(\sigma) \subset X(\Delta)$. If $\sigma_{1}, \ldots, \sigma_{k}$ denote the minimal cones of $\Delta^{\prime}$ such that $\sigma$ is minimal over $\phi\left(\sigma_{i}\right)$ then the irreducible decomposition of the inverse image 
of $V(\sigma)$ is:

$$
\varphi^{-1}(V(\sigma))=\bigcup_{i=1}^{k} V\left(\sigma_{i}\right) .
$$

In particular, in the case of a refinement, $\sigma_{i}$ can be characterized as the minimal cones intersecting the relative interior of $\sigma$.

There is a special refinement which will be important in the remainder of the paper - the star subdivision. Geometrically, this corresponds to the blowup of $X(\Delta)$ at a distinguished point $\gamma_{\sigma}$. Specifically, given a cone $\sigma$, let $u_{1}, \ldots, u_{k}$ be the primitive generators of the rays of $\sigma$, then the star of $\sigma$ is the vector $\operatorname{Star}(\sigma)=\sum_{i=1}^{k} u_{i}$. The star subdivision of $\sigma$, see [CLS, Definition 3.3.13], is the fan denoted $\sigma^{*}$ whose maximal cones are all of the form $\operatorname{Cone}(\mu, \operatorname{Star}(\sigma))$ where $\mu \subset \sigma$ is a facet.

As a final remark, recall that a cone is smooth if the minimal generators of the rays form part of an integral basis of $N$, and that a cone is simplicial if the minimal generators are linearly independent over $\mathbb{R}$. A fan is smooth or simplicial if each of its cones are smooth or simplicial respectively. It is well known that the toric variety $X(\Delta)$ is smooth if and only if each cone in $\Delta$ is smooth.

2.2. Cox Construction of a Toric Variety. The Cox construction produces any toric variety as the good quotient of an open subscheme of affine space by the action of a diagonalizable group. Following [CLS, Chapter 5], we briefly recall the salient details of the construction. Given a full-dimensional fan $\Delta$ on a lattice $N$, the total coordinate ring of $X(\Delta)$ is the polynomial ring $k\left[x_{\rho}: \rho \in \Delta(1)\right]$ freely generated by the variables $x_{\rho}$. Suppose $n=|\Delta(1)|$ such that $\mathbb{A}^{n}=\operatorname{Spec}\left(k\left[x_{\rho}\right]\right)$, then for each cone $\sigma \in \Delta$, define the monomial:

$$
x^{\sigma}=\prod x_{\rho_{i}} \text { where }\left\{\rho_{i}\right\} \text { are the rays not contained in } \sigma .
$$

Furthermore, define $B(\Delta)=\left(x^{\sigma}\right)_{\sigma \in \Delta}$ to be the ideal generated by these monomials. The exceptional set of $\Delta$ is the following closed subscheme of $\mathbb{A}^{n}$ :

$$
Z(\Delta)=V(B(\Delta)) \subset \mathbb{A}^{n} \text {. }
$$

The closed set $Z(\Delta)$ has an explicit, combinatorial description. Recall that a primitive collection of rays is a collection of rays satisfies the following two properties: the collection is not contained in a single cone of $\Delta$, but every proper subset is contained in a cone of $\Delta$. For each primitive collection of rays $\left\{\rho_{i}\right\}$, we can generate a linear subspace given by $V\left(\left(x_{\rho_{i}}\right)_{i \in I}\right)$.

Proposition 2.2. CLS, Proposition 5.1.6] The irreducible components of $Z(\Delta)$ are in bijection with the linear subspaces $\mathbb{V}\left(\left(x_{\rho_{i}}\right)_{i \in I}\right)$ where $I$ is a primitive collection of rays.

Given a fan $\Delta$, let $\Sigma_{\Delta}$ denote the fan generated by cones $\bar{\sigma}=\operatorname{Cone}\left(e_{\rho}: \rho \in \sigma(1)\right) \subset \mathbb{R}^{|\Delta(1)|}$ where $\sigma$ runs through the cones of $\Delta$.

Proposition 2.3. [CLS, Proposition 5.1.9] (a) $X\left(\Sigma_{\Delta}\right)=\mathbb{A}^{n} \backslash Z(\Delta)$.

(b) The map $e_{\rho} \mapsto u_{\rho}$ defines a map of lattices $\mathbb{Z}^{\Sigma(1)} \rightarrow N$ that is compatible with the fans $\Sigma_{\Delta} \subset \mathbb{R}^{\Delta(1)}$ and $\Delta \subset N_{\mathbb{R}}$.

Remark 2.4. Note that if $\sigma$ is a cone of $\Delta$, then the orbit closure $V(\sigma)$ is the image of the linear subspace $\mathbb{V}\left(\left\{x_{\rho}\right\}_{\rho \in \sigma(1)}\right)$. 
The diagonalizable group $G$ is constructed as follows: suppose $\operatorname{dim}(\Delta)=k$, then the morphism of lattices $\mathbb{Z}^{n} \rightarrow \mathbb{Z}^{k}$ defined by sending the canonical basis vectors to the minimal generators of rays of $\Delta$ has finite cokernel; hence, the dual map is injective, and we set $L$ to be the cokernel of this map. The group $G$ is defined to be $\operatorname{Hom}\left(L, \mathbb{G}_{m}\right)$, the group of characters of $L$, which injects into the torus of $\mathbb{A}^{n}$.

Theorem 2.5. CLS, Theorem 5.1.11(a)] Under the above notation, the toric map $\left(\mathbb{A}^{n} \backslash Z(\Delta)\right) \rightarrow X(\Delta)$ identifies $X(\Delta)$ as the good quotient $\left(\mathbb{A}^{n} \backslash Z(\Delta)\right) / G$.

2.3. Canonical Toric Stacks. Given a full-dimensional fan $\Delta$ on a lattice $N$ and its associated toric variety $X(\Delta)$, the canonical toric stack is a smooth quotient stack possessing a special relationship with $X(\Delta)$. Following [GS, Section 5], there are two equivalent definitions for the canonical stack. The first is given in terms of the Cox construction, and the second constructs $\mathcal{X}(\Delta)$ as a toric stack arising from a canonical stacky fan. The theory of stacky fans is worked out in [GS] and provides a combinatorial gadget for toric stacks analogous to fans for toric varieties. A stacky fan is a pair $(\Delta, \beta: N \rightarrow L)$ where $\Delta$ is a lattice on $N$ and $\beta$ is a morphism of lattices with finite cokernel, and $\beta$ induces a surjective morphism of tori $T_{N} \rightarrow T_{L}$ with kernel $G_{\beta}$. The toric stack associated to this data is the quotient stack $\left[X(\Delta) / G_{\beta}\right]$ with torus $T_{L}$.

Following [GS, Section 3], toric morphisms between toric stacks arising from stacky fans $\left(\Delta^{\prime}, N^{\prime} \rightarrow L^{\prime}\right),(\Delta, N \rightarrow L)$ correspond to morphisms of lattices which are compatible with the stacky fans: specifically, $\phi: N^{\prime} \rightarrow N$ and $\Phi: L^{\prime} \rightarrow L$ are morphisms of lattices such that for every cone $\sigma \in \Delta^{\prime}, \phi(\sigma)$ is contained in a cone of $\Delta$ and the following diagram commutes:

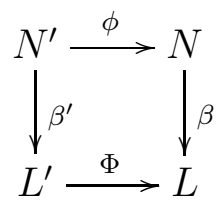

Definition 2.1. The canonical stack of $X(\Delta)$ will be denoted by $\mathcal{X}(\Delta)$ and is equipped with a toric morphism $\mathcal{X}(\Delta) \rightarrow X(\Delta)$; it can be constructed in either of the following ways:

(1) The Cox construction of a toric variety produces an open subscheme $U \subset \mathbb{A}^{|\Delta(1)|}$ and a diagonalizable group $G$ such that $X(\Delta) \cong U / G$, CLS, Theorem 5.1.11]. The canonical stack of $X(\Delta)$ is the quotient stack $[U / G]$ with morphism $[U / G] \rightarrow X(\Delta)$.

(2) Equivalently, let $\left(\Sigma_{\Delta}, \beta: \mathbb{Z}^{|\Delta(1)|} \rightarrow N\right)$ be the stacky fan where $\beta$ is defined by sending the canonical basis vectors to the primitive generators of $\Delta$, and $\Sigma_{\Delta}$ is the canonical fan defined in the previous section. The toric stack associated to this stacky fan is the canonical stack $\mathcal{X}(\Delta)$.

Furthermore, the toric morphism $\mathcal{X}(\Delta) \rightarrow X(\Delta)$ is easily constructed from the following morphism of stacky fans:

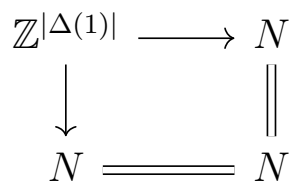

Recall Alp, Definition 4.1], that a morphism from an Artin stack to an algebraic space is a good moduli space morphism if it is cohomologically affine and Stein. In the context of quotient stacks, this is a natural generalization of good quotients. As a consequence of [GS, Theorem 6.3], the following holds: 
Theorem 2.6. [GS, Example 6.23] The canonical stack morphism $\mathcal{X}(\Delta) \rightarrow X(\Delta)$ is a good moduli space morphism.

Example 2.7. Let $\sigma$ denote the cone generated by $\{(1,0,1),(0,-1,1),(-1,0,1),(0,1,1)\}$ in $\mathbb{R}^{3}$, then $X(\sigma)$ is a 3 -dimensional, affine, singular toric variety with four rays. Let $\beta: \mathbb{Z}^{4} \rightarrow \mathbb{Z}^{3}$ be defined by sending the canonical basis vectors to the primitive generators of $\sigma$; note, the preimage of $\sigma$ under $\beta$ is the cone defining $\mathbb{A}^{4}$. Furthermore, the cokernel of the dual of $\beta$ is $\mathbb{Z}^{4} \rightarrow \mathbb{Z}^{3}$ defined as the matrix $[1,-1,1,-1]$; hence, if we apply $D(-):=\operatorname{Hom}\left(-, \mathbb{G}_{m}\right)$ to this cokernel, we see that $G_{\beta}=\mathbb{G}_{m}$ and defines an action of $\mathbb{A}^{4}$ with weights $(1,-1,1,-1)$. Thus, the canonical stack of $X(\sigma)$ is $\mathcal{X}(\sigma)=\left[\mathbb{A}^{4} / \mathbb{G}_{m}\right]$.

\section{Chow Cohomology of Algebraic Spaces and Stacks}

Following [Ful1], let $A_{k}(X)$ denote the group of dimension $k$ cycle classes modulo rational equivalence, and if $X$ is equidimensional, let $A^{k}(X)$ denote the group of codimension $k$ cycle classes modulo rational equivalence. In addition if $X$ is smooth, then the intersection product on $A_{k}(X)$ as constructed in [Ful1, Chapter 6.1] makes $A^{*}(X)$ into a commutative, graded ring. For general schemes, without any assumptions on non-singularity, [Ful1, Chapter 17] constructs a graded ring $A_{\mathrm{op}}^{*}(X):=\oplus_{k \geq 0} A_{\mathrm{op}}^{k}(X)$ defined as follows: an element $c \in A_{\mathrm{op}}^{k}(X)$ is a collection of homomorphisms of groups:

$$
c_{g}^{(k)}: A_{p} X^{\prime} \rightarrow A_{p-k} X^{\prime}
$$

for every morphism $g: X^{\prime} \rightarrow X$ which are compatible with respect to proper pushforward, and flat and l.c.i. pullbacks (see [Ful1, Definition's 17.1 and 17.3]). The product is given by composition and turns $A_{\mathrm{op}}^{*}(X)$ into a unital, associative, graded ring called the Chow cohomology ring of $X$. Moreover, if $X$ has a resolution of singularities (e.g. if $X$ is a toric variety) then $A_{\mathrm{op}}^{*}(X)$ is known to be commutative. If $X$ is smooth, then [Ful1, Corollary 17.4] proves that the Poincaré-Duality map $A_{\mathrm{op}}^{k}(X) \rightarrow A^{k}(X)=A_{n-k}(X)$ is an isomorphism of rings wherein the intersection product agrees with the product given by composition.

Remark 3.1. In [Ful1, Chapter 17] the Chow cohomology ring is also denoted $A^{*}(X)$ without the inclusion of the subscript 'op', regardless if $X$ is smooth or not. We will deviate from this convention to emphasize the distinction between operational classes in the smooth and non-smooth cases.

For smooth quotient stacks $\mathcal{X}=[Z / G]$, the Chow ring $A^{*}(\mathcal{X})$ is identified with the $G$ equivariant Chow ring $A_{G}^{*}(Z)$ by [EG]. Furthermore, if $\pi: \mathcal{X} \rightarrow X$ is any morphism between a stack and an algebraic space, [ES, Proposition 2.10] proves that there is a a pullback map $\pi^{*}: A_{\mathrm{op}}^{*}(X) \rightarrow A^{*}(\mathcal{X}), c \mapsto c \cap[\mathcal{X}]$. In this paper we make essential use of the following result.

Theorem 3.2. [ES, Theorem 1.1] Let $\mathcal{X}$ be a smooth, connected, properly stable, Artin stack with good moduli space $\pi: \mathcal{X} \rightarrow X$, then $\pi^{*}$ is injective over $\mathbb{Q}$.

Since $\mathcal{X}(\Delta) \rightarrow X(\Delta)$ is a good moduli space morphism, we conclude that $A_{\mathrm{op}}^{*}(X(\Delta))_{\mathbb{Q}}$ injects into $A^{*}\left(\mathcal{X}(\Delta)_{\mathbb{Q}}\right.$. If $\Delta$ is simplicial, then $\mathcal{X}(\Delta)$ is Deligne-Mumford and the inclusion is an isomorphism. 
3.1. Chow Cohomology of Toric Varieties. For toric varieties, the generators and relations of the Chow groups $A_{k}(X(\Delta))$ can be explicitly computed. By [Ful2, Chapter 5.1], $A_{k}(X(\Delta))$ is generated by the classes $[V(\sigma)]$ where $\sigma$ has codimension $k$. Furthermore, the group of relations between these generators is generated by all relations of the form:

$$
\left[\operatorname{div}\left(x^{u}\right)\right]=\sum_{\sigma}\left\langle u, n_{\sigma, \tau}\right\rangle[V(\sigma)]
$$

where $\tau$ runs over all cones of codimension $k+1$ which are a face of $\sigma, u$ runs over a generating set of $M(\tau)$, and $n_{\sigma, \tau}$ is a rational point whose image generates the lattice $N_{\sigma} / N_{\tau}$. In the case that $X(\Delta)$ is smooth, the above description can be used to compute $A_{\mathrm{op}}^{*}(X(\Delta))$ since $A_{\text {op }}^{k}(X(\Delta))=A_{n-k}(X(\Delta)$. However when $X(\Delta)$ is not smooth, there are only a few known techniques to compute $A_{\mathrm{op}}^{*}(X(\Delta))$. In particular, for complete toric varieties, the Chow cohomology ring can also be derived from the above description of Chow groups by the following theorem:

Theorem 3.3. [FS, Proposition 1.4, Theorem 2.1] If $X$ is a complete toric variety, then $A_{\mathrm{op}}^{k}(X) \cong \operatorname{Hom}\left(A_{k}(X), \mathbb{Z}\right)$, and the Chow cohomology is also isomorphic to the ring of Minkowski weights on $\Delta$.

However, for non-complete toric varieties this isomorphism fails as the following example demonstrates.

Example 3.4. Let $\sigma$ denote the cone generated by $\{(1,0,1),(0,-1,1),(-1,0,1),(0,1,1)\}$ in $\mathbb{R}^{3}$ as seen above. One can compute that $A_{2}(X(\sigma))=\mathbb{Z} / 2 \oplus \mathbb{Z}$ which does admit non-trivial morphisms $A_{2}(X(\sigma)) \rightarrow \mathbb{Z}$. However, by Theorem 4.1, $A_{\text {op }}^{2}(X(\sigma))=0$.

3.1.1. Computing Chow Cohomology: Kimura's Exact Sequence. There is a second technique to compute the Chow cohomology of a toric variety using Kimura's exact sequence. This sequences injects $A_{\mathrm{op}}^{*}(X(\Delta))$ into the Chow cohomology of any envelope of $X(\Delta)$. Recall, a morphism $X^{\prime} \rightarrow X$ is an envelope if it is proper and for every closed subvariety $V \subset X$, there exists a closed subvariety $V^{\prime} \subset X^{\prime}$ mapping onto $V$ such that the restriction $V^{\prime} \rightarrow V$ is birational.

Proposition 3.5. [Kim, Theorem 2.3] Let $X^{\prime} \rightarrow X$ be an envelope which is an isomorphism outside of $S \subset X$ and $E \subset X^{\prime}$, then

$$
0 \rightarrow A_{\mathrm{op}}^{*}(X) \rightarrow A_{\mathrm{op}}^{*}\left(X^{\prime}\right) \oplus A_{\mathrm{op}}^{*}(S) \rightarrow A_{\mathrm{op}}^{*}(E)
$$

is exact.

If $X^{\prime} \rightarrow X$ is proper and surjective, then the above sequence is exact after tensoring with $\mathbb{Q}$. Payne proved [Pay, Lemma 1] that any proper birational, toric morphism is an envelope. Hence, if $\Delta^{\prime}$ is any refinement of $\Delta$, then $A_{\mathrm{op}}^{*}(X(\Delta))$ injects into $A_{\mathrm{op}}^{*}\left(X\left(\Delta^{\prime}\right)\right)$. We make essential use of this fact in our proof of Theorem 4.1

3.2. Chow Rings of Canonical Toric Stacks. The Chow ring of $\mathcal{X}(\Delta)$ admits a simple presentation using equivariant Chow groups. Following [CE, Section 2], let $n=|\Delta(1)|$, $V=\mathbb{A}^{n}, U=V \backslash Z(\Delta), G$ the diagonalizable group such that $\mathcal{X}(\Delta)=[U / G]$ and $X(G)$ the character group of $G$. The excision exact sequence ([Ful1, Prop. 1.8]) for equivariant Chow groups implies $A^{*}(\mathcal{X}(\Delta))$ is a quotient of $A_{G}^{*}(V)=A_{G}^{*}(p t)$; the latter $A_{G}^{*}(p t)$ will be denoted by $A_{G}^{*}$. By $[\mathrm{EG}], A_{G}^{*} \cong \mathbb{Z}[X(G)]$ where the latter is the symmetric algebra of the character 
group of $G$. By construction of $\mathcal{X}(\Delta)$, the action of $G$ on $V$ is faithful; hence, $G$ injects into $\mathbb{G}_{m}^{n}$ and induces a surjection of character groups $X\left(\mathbb{G}_{m}^{n}\right) \rightarrow X(G)$. This surjection further induces a surjection of equivariant Chow rings $A_{\mathbb{G}_{m}^{n}}^{*} \rightarrow A_{G}^{*}$ leading us to the following definition:

Definition 3.1. The linear equivalence ideal of $\Delta$ is defined to be:

$$
L(\Delta):=\operatorname{ker}\left(\mathbb{A}_{\mathbb{G}_{m}^{n}}^{*} \rightarrow A_{G}^{*}\right)
$$

Equivalently, it is the linear ideal generated by the relations among the image of $X\left(\mathbb{G}_{m}^{n}\right)$ in $X(G)$. Hence, $A_{G}^{*}=A_{\mathbb{G}_{m}^{n}}^{*} / L(\Delta)$.

Suppose $L_{1}, \ldots, L_{k}$ are the irreducible components of $Z(\Delta)$, then by [CE, Propositions 2.1 and 2.2], $A_{G}^{*}(U)$ is the quotient of $A_{\mathbb{G}_{m}^{n}}^{*}$ modulo the linear equivalence ideal and the ideal generated by the equivariant fundamental classes of $L_{i}$.

Definition 3.2. The Stanley-Reisner ideal of $\Delta$ is the ideal $\mathcal{Z}(\Delta)$ generated by the equivariant fundamental classes of the irreducible components of $Z(\Delta)$.

Thus, by [CE, Propositions 2.1 and 2.2], the Chow ring of $\mathcal{X}(\Delta)$ has the following presentation:

Theorem 3.6. Under the above notation,

$$
A^{*}(\mathcal{X}(\Delta)) \cong A_{G}^{*} / \mathcal{Z}(\Delta) \cong A_{\mathbb{G}_{m}^{n}}^{*} /(L(\Delta), \mathcal{Z}(\Delta))
$$

Each of the terms in the above presentation can be explicitly computed. Let $e_{1}, \ldots, e_{n}$ denote the canonical basis characters in $X\left(\mathbb{G}_{m}^{n}\right)$, and let $t_{1}, \ldots, t_{n}$ denote the corresponding first Chern classes of the associated 1-dimensional representations of each character, then $A_{\mathbb{G}_{m}^{n}}^{*}=$ $\mathbb{Z}\left[t_{1}, \ldots, t_{n}\right]$. For the linear equivalence ideal, let $v_{1}, \ldots, v_{k}$ be a basis for $\operatorname{ker}\left(X\left(\mathbb{G}_{m}^{n}\right) \rightarrow X(G)\right)$, then $L(\Delta)$ is the ideal generated by corresponding first Chern classes of $v_{1}, \ldots, v_{k}$ : if $v_{i}=$ $\sum_{j} a_{i j} e_{j}$, then $\sum_{j} a_{i j} t_{j} \in L(\Delta)$. For the Stanley-Reisner ideal, suppose $L_{i}=V\left(x_{i_{1}}, \ldots, x_{i_{l}}\right)$ where $x_{1}, \ldots, x_{n}$ are coordinates for $V$, then the equivariant fundamental class of $L_{i}$ is $t_{i_{1}} \cdot \ldots \cdot t_{i_{l}}$.

Remark 3.7. Since we know the irreducible components of $Z(\Delta)$ correspond to the primitive collections of $\Delta, \mathcal{Z}(\Delta)$ is equivalently generated by the monomials corresponding to primitive collections. Explicitly, if $\left\{\rho_{i_{1}}, \ldots, \rho_{i_{k}}\right\}$ is a primitive collection corresponding to an irreducible component of $Z(\Delta)$, then the monomial $t_{i_{1}} \cdot \ldots \cdot t_{i_{k}}$ is the equivariant fundamental class of this component.

Example 3.8. Let $\sigma$ be the cone in the previous examples, then since $X(\sigma)$ is affine, $Z(\sigma)=$ 0 . Furthermore, $L(\sigma)$ is generated by the relations $\left(s_{1}-s_{3}, s_{2}-s_{4}, s_{1}+\ldots+s_{4}\right)$. Hence, $A^{*}(\mathcal{X}(\sigma))$ is a polynomial ring in one variable.

Example 3.9. Suppose we star subdivide $\sigma$ to produce the fan $\sigma^{*}$. The Chow cohomology of the canonical stack of $\sigma^{*}$ has the following presentation: the Stanley-Reisener ideal is the ideal $\left(s_{1} s_{3}, s_{2} s_{4}\right)$ since $\left\{\rho_{1}, \rho_{3}\right\}$ and $\left\{\rho_{2}, \rho_{4}\right\}$ are the primitive collections of $\sigma^{*}$. Furthermore, the linear equivalence ideal is defined by the equations $\left(s_{1}, s_{3}, s_{2}-s_{4}, s_{1}+\ldots+s_{5}\right)$. Hence, $A^{*}\left(\mathcal{X}\left(\sigma^{*}\right)\right)=\mathbb{Z}\left[s_{1}, s_{2}\right] /\left(s_{1}^{2}, s_{2}^{2}\right)$.

\section{Chow Cohomology of Affine Toric Varieties}

In this section, we will establish our main result, the vanishing of the Chow cohomology of an affine toric variety. 
Theorem 4.1. For any affine toric variety $X(\sigma)$,

$$
A_{\mathrm{op}}^{k}(X(\sigma))=0 \text { for } k>0 .
$$

In particular, $A_{\mathrm{op}}^{*}(X(\sigma))=\mathbb{Z}$.

To slightly simplify the notation we will assume that the cone is full dimensional. If $\sigma$ is not full dimensional then $X(\sigma)=T \times X(\bar{\sigma})$ where $T$ is a torus and $\bar{\sigma}$ is a full-dimensional cone and our full-dimensional proof readily adapts to this case.

The proof will be divided into the following subsections.

4.1. Kimura's Exact Sequence. Let $\sigma^{*}$ denote the star subdivision of $\sigma$ with respect to its star, $v=\operatorname{Star}(\sigma), \rho_{v}$ the corresponding ray in $\sigma^{*}$, and $\phi: X\left(\sigma^{*}\right) \rightarrow X(\sigma)$ the associated toric morphism. Note, this morphism is an isomorphism outside of $S:=V(\sigma)$ and $E:=\phi^{-1}(S)$.

Lemma 4.2. Under the above notation, $E=V\left(\rho_{v}\right)$.

Proof. By construction, $\rho_{v}$ is contained in the interior of $\sigma$, and if $\tau$ is a cone of $\sigma^{*}$ nontrivially intersecting the interior of $\sigma$, then $\tau$ necessarily contains $\rho_{v}$. Hence, $\rho_{v}$ is the unique minimal cone of $\sigma^{*}$ intersecting the interior of $\sigma$. The Lemma then follows from the description of Theorem 2.1.

Furthermore, $S$ is 0-dimensional and irreducible, hence, $A^{0}(S)=\mathbb{Z}$ and $A^{k}(S)=0$ for $k>0$. Therefore, if we apply Kimura's exact sequence to $\phi$, we obtain the following exact sequence:

$$
0 \rightarrow A_{\mathrm{op}}^{k}(X(\sigma)) \rightarrow A_{\mathrm{op}}^{k}\left(X\left(\sigma^{*}\right)\right) \rightarrow A_{\mathrm{op}}^{*}\left(V\left(\rho_{v}\right)\right) \text { for } k>0 .
$$

Thus, to show $A_{\mathrm{op}}^{k}(X(\sigma))=0$ for $k>0$, it suffices to show $A_{\mathrm{op}}^{k}\left(X\left(\sigma^{*}\right)\right) \rightarrow A_{\mathrm{op}}^{*}\left(V\left(\rho_{v}\right)\right)$ is injective. To accomplish this, Steps 2 and 3 will further demonstrate that it suffices to consider the Chow rings of the associated canonical stacks. In Step 4 we will show that these Chow rings are in fact isomorphic.

4.2. A Commutative Diagram of Canonical Stacks. Let $\mathcal{X}\left(\sigma^{*}\right) \rightarrow X\left(\sigma^{*}\right)$ and $\mathcal{E} \rightarrow$ $E$ denote the canonical stacks of $X\left(\sigma^{*}\right)$ and $E$ respectively, and let $E \rightarrow X\left(\sigma^{*}\right)$ denote the inclusion, then the goal of this subsection is to show that we can construct a closed embedding $\mathcal{E} \rightarrow \mathcal{X}\left(\sigma^{*}\right)$ such that the following diagram of canonical stacks and moduli spaces is commutative:

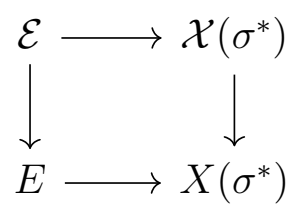

Let $\Sigma_{\sigma^{*}}$ denote the fan of $\mathbb{A}^{\left|\sigma^{*}(1)\right|} \backslash Z\left(\sigma^{*}\right)$. It is equipped with a morphism $X\left(\Sigma_{\sigma^{*}}\right) \rightarrow X\left(\sigma^{*}\right)$ as in the canonical stack construction. If $n=|\sigma(1)|$, let $x_{1}, \ldots, x_{n+1}$ be coordinates on $\mathbb{A}^{\left|\sigma^{*}(1)\right|}$ such that $x_{n+1}$ corresponds to the ray $\rho_{v}$. By Remark 2.4 we have a commutative diagram of $G$-quotients 


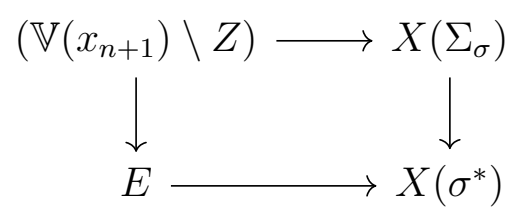

where $G$ is the group acting on $X\left(\Sigma_{\sigma^{*}}\right)$ such that $\mathcal{X}\left(\sigma^{*}\right)=\left[X\left(\Sigma_{\sigma^{*}}\right) / G\right]$. We will show that $\left[\left(\mathbb{V}\left(x_{n+1}\right) \backslash Z / G\right)\right]$ is the canonical stack of the toric variety $E$.

We begin with the following basic result of toric varieties:

Lemma 4.3. A collection of rays of $\sigma^{*}$ is primitive if and only if the corresponding collection in $\operatorname{Star}\left(\rho_{v}\right)$ is primitive.

Proof. Let $C$ be a primitive collection of $\sigma^{*}$ and and let $C^{\prime}$ be the associated collection in $\operatorname{Star}\left(\rho_{v}\right)$. If $D^{\prime} \subset C^{\prime}$ is a proper subset and $D \subset C$ denotes the corresponding subset, then since $D$ is contained in some cone, it follows that $D^{\prime}$ will be contained in the image of that cone, and similarly, if $C^{\prime} \subset \bar{\tau}(1)$, then $C \subset \tau$ would contradict $C$ being primitive. Thus, if $C$ is primitive, then $C^{\prime}$ is primitive; the converse holds by a similar argument.

The existence of the commutative diagram (2) now follows from the following lemma.

Lemma 4.4. Let $\mathcal{E}$ denote the canonical stack over $E$, then $\mathcal{E} \cong\left[\left(\mathbb{V}\left(x_{n+1}\right) \backslash Z\right) / G\right]$.

Proof. By definition, $\mathcal{E}$ is the quotient stack of the form $\left[\left(\mathbb{A}^{n} \backslash Z^{\prime}\right) / G^{\prime}\right]$ where $Z^{\prime}$ and $G^{\prime}$ are defined as previously. By the previous lemma, there is an obvious isomorphism between $\mathbb{A}^{n} \backslash Z^{\prime}$ and $\mathbb{V}\left(x_{n+1}\right) \backslash Z\left(\sigma^{*}\right)$. We claim that $G=G^{\prime}$ and the action of $G^{\prime}$ on $\mathbb{A}^{n} \backslash Z^{\prime}$ coincides with the action of $G$ on $\mathbb{V}\left(x_{n+1}\right) \backslash Z\left(\sigma^{*}\right)$ under this isomorphism. Indeed, this follows from the commutativity of the following diagram of character groups:

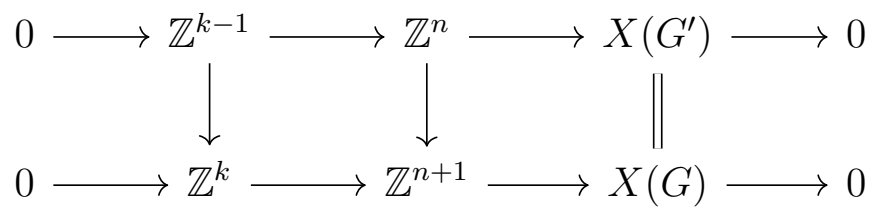

The vertical maps are the duals of the canonical quotient maps for the primitive generators of $\rho_{v}$ and $e_{n+1}$ respectively.

Remark 4.5. Note that unless $\sigma^{*}$ is a resolution of singularities, diagram (3) is not cartesian. The following examples shows that even if $\sigma^{*}$ is a simplicialization the diagram fails to be Cartesian.

Example 4.6. Consider the cone $\sigma$ with ray generators given by $\{(1,1,1),(-1,0,1),(1,0,1)$, $(0,-1,1)\}$, and its associated star subdivision $\sigma^{*}$ obtained by adding the ray generator $(1,0,4)$ and subdividing appropriately. We claim that in this example, $\mathcal{E}$ is not schemetheoretically saturated with respect to $\pi$. Following [ES, Remark 3.4], since $\operatorname{dim}(\mathcal{E})=$ $\operatorname{dim}(E)$ and $\pi(\mathcal{E})=E$, it suffices to show $\mathcal{E}$ is not a strong divisor.

With the notation as in this section, the action of $\mathbb{G}_{m}^{2}$ on $\mathbb{A}^{5} \backslash V\left(x_{1}, x_{3}\right) \cup V\left(x_{2}, x_{4}\right)$ is given by the matrix:

$$
\left[\begin{array}{lllll}
3 & -2 & 1 & -2 & 0 \\
2 & -3 & 0 & -3 & 1
\end{array}\right]
$$


If $\mathcal{E}$ is strong, then the ideal $\left(x_{5}\right)$ is generated by an invariant function on each open set $D\left(x_{1} x_{2}\right), D\left(x_{2} x_{3}\right), D\left(x_{3} x_{4}\right)$, and $D\left(x_{1} x_{4}\right)$. However, on $D\left(x_{1} x_{2}\right)$ where $x_{1}, x_{2}$ are invertible, the weight $(0,1)$ of $x_{5}$ cannot be expressed as integral linear combination of the weights $(3,2)$ and $(-2,-3)$ for $x_{1}, x_{2}$ respectively; thus, $\mathcal{E}$ is not strong. Note that $(0,1)$ can be expressed rationally in terms of these weights on each affine open set and it is easy to check that $\left(x_{5}^{15}\right)$ is locally generated by an invariant function.

4.3. The injectivity result of [ES]. Since the canonical stack morphisms are good moduli space morphisms, we have that $A_{\mathrm{op}}^{*}(E)_{\mathbb{Q}} \subset A^{*}(\mathcal{E})_{\mathbb{Q}}$ and $A_{\mathrm{op}}^{*}\left(X\left(\sigma^{*}\right)\right)_{\mathbb{Q}} \subset A^{*}\left(\mathcal{X}\left(\sigma^{*}\right)_{\mathbb{Q}}\right.$. Hence, by combining these injections with the main result of the previous section and Kimura's exact sequence, the following diagram commutes:

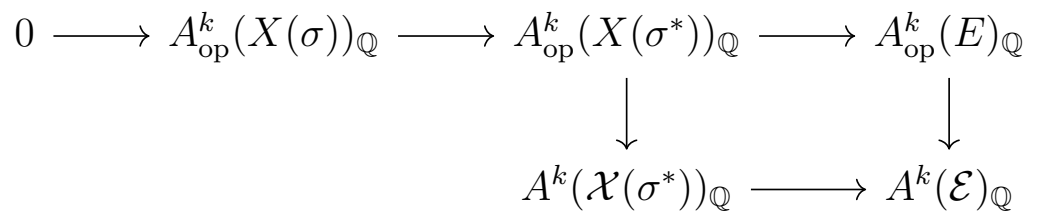

Since the vertical maps are injections, to show $A_{\mathrm{op}}^{k}\left(X\left(\sigma^{*}\right)\right) \rightarrow A_{\mathrm{op}}^{k}(E)$ is injective at least over $\mathbb{Q}$, it suffices to show $A^{*}\left(\mathcal{X}\left(\sigma^{*}\right)\right)_{\mathbb{Q}} \rightarrow A^{*}(\mathcal{E})_{\mathbb{Q}}$ is an injection. In fact, we claim this map is an isomorphism over $\mathbb{Z}$.

\subsection{The Pullback of Chow Rings of Canonical Stacks is an Isomorphism.}

Theorem 4.7. Let $\mathcal{E} \rightarrow \mathcal{X}\left(\sigma^{*}\right)$ be the injective morphism constructed in Step 2, then the pullback induces an isomorphism $A^{*}(\mathcal{E}) \cong A^{*}\left(\mathcal{X}\left(\sigma^{*}\right)\right)$.

Proof. By the previous lemma, $\mathcal{E} \cong\left[\left(\mathbb{V}\left(x_{n+1}\right) \backslash Z\left(\sigma^{*}\right)\right) / G\right] \subset \mathcal{X}\left(\sigma^{*}\right)=\left[\left(\mathbb{A}^{n+1} \backslash Z\left(\sigma^{*}\right)\right) / G\right]$. Hence, the Chow rings of the canonical stacks are the following equivariant Chow rings:

$$
A^{*}\left(\mathcal{X}\left(\sigma^{*}\right)\right)=A_{G}^{*}\left(\mathbb{A}^{n+1} \backslash Z\left(\sigma^{*}\right)\right) \text {, and } A^{*}(\mathcal{E})=A_{G}^{*}\left(V\left(x_{n+1}\right) \backslash Z\left(\sigma^{*}\right)\right) .
$$

Since $Z\left(\sigma^{*}\right)$ does not contain $x_{n+1}$, we can write $\mathbb{V}\left(x_{n+1}\right) \backslash Z\left(\sigma^{*}\right)=\mathbb{A}^{n} \backslash Z^{\prime}$ and $\mathbb{A}^{n+1} \backslash Z\left(\sigma^{*}\right)=$ $\left(\mathbb{A}^{n} \backslash Z^{\prime}\right) \times \mathbb{A}^{1}$ for the obvious exceptional set $Z^{\prime} \subset \mathbb{A}^{n}$. In particular, the inclusion of canonical stacks is the quotient by $G$ of the zero section $\mathbb{A}^{n} \backslash Z^{\prime} \subset\left(\mathbb{A}^{n} \backslash Z^{\prime}\right) \times \mathbb{A}^{1}$ of a $G$-equivariant vector bundle over $\mathbb{A}^{n} \backslash Z^{\prime}$. Hence, the pullback in $G$-equivariant Chow groups along this inclusion is an isomorphism.

Thus, $A_{\mathrm{op}}^{k}(X(\sigma))_{\mathbb{Q}}=0$ for $k>0$. The final step will be to show $A_{\mathrm{op}}^{k}(X(\sigma))$ is torsion-free.

4.5. Chow Cohomology of Semi-Projective Toric Varieties is Torsion-Free. From $[\mathrm{HS}$, a toric variety $X(\Delta)$ is semi-projective if it has at least one torus fixed point and the natural map $X(\Delta) \rightarrow \operatorname{Spec}\left(\Gamma\left(\mathcal{O}_{X(\Delta)}\right)\right)$ is projective; equivalently, it is semi-projective if $\Delta$ has full-dimensional convex support and $X(\Delta)$ is quasi-projective. Hence, for an affine toric variety $X(\sigma)$, any refinement $\Delta$ of $\sigma$ produces a semi-projective toric variety $X(\Delta)$ which is projective over $X(\sigma)$. In particular, let $\sigma^{\prime}$ be a resolution of singularities of $\sigma$, then by $\underline{\mathrm{Kim}}$, Lemma 2.1], $A_{\mathrm{op}}^{*}(X(\sigma)) \subset A_{\mathrm{op}}^{*}\left(X\left(\sigma^{\prime}\right)\right)$. Hence, it suffices to show $A_{\mathrm{op}}^{*}\left(X\left(\sigma^{\prime}\right)\right)$ is torsion-free where $X\left(\sigma^{\prime}\right)$ is a smooth semi-projective toric variety.

Remark 4.8. For a smooth projective toric variety, [Ful2, Section 5.2] demonstrates that its Chow ring is torsion-free by producing Bialynicki-Birula decomposition from a particular ordering of the maximal dimensional cones. 
In [HS, Section 2], Hausel and Sturmfels generalize the construction of [Ful2, Section 5.2] to smooth semi-projective toric varieties by utilizing the moment map associated to a 1parameter subgroup, and in particular, they construct the Bialynicki-Birula decomposition of a smooth semiprojective variety by producing a collection of locally closed subsets $\left\{U_{j}\right\}$ satisfying the following properties:

(1) Each $U_{i}$ is a union of orbits, and hence, the closure of $U_{i}$ is a union of $U_{j}$ 's.

(2) $X\left(\sigma^{\prime}\right)$ is the disjoint union of the $U_{i}$ 's.

(3) Each $U_{i} \cong \mathbb{A}^{n-k_{i}}$.

In particular, by [EH, Definition 1.16], this decomposition of $X\left(\sigma^{\prime}\right)$ is an affine stratification. By [Tot], the classes of $U_{i}$ form a basis for $A^{*}\left(X\left(\sigma^{\prime}\right)\right)$, and hence, $A^{*}\left(X\left(\sigma^{\prime}\right)\right)$ is necessarily torsion-free. Thus, since $A_{\mathrm{op}}^{*}(X(\sigma)) \subset A^{*}\left(X\left(\sigma^{\prime}\right)\right)$, it follows that $A_{\mathrm{op}}^{*}(X(\sigma))$ is torsion-free, and by combining all of the previous subsections, this concludes the proof of the main theorem.

The argument presented in this subsection can be utilized to demonstrate that the Chow cohomology rings of a wide class of toric varieties are torsion-free. As a natural generalization of semi-projectivity, we say that a toric variety $X(\Delta)$ is semi-proper if it has at least one torus fixed point and the natural map $X(\Delta) \rightarrow \operatorname{Spec}\left(\Gamma\left(\mathcal{O}_{X(\Delta)}\right)\right)$ is proper.

Theorem 4.9. Let $X(\Delta)$ be any semi-proper toric variety, then $A_{\mathrm{op}}^{*}(X(\Delta))$ is torsion-free.

Proof. By the toric Chow's lemma [CLS, Theorem 6.1.18], there exists a projective toric variety $X\left(\Delta^{\prime}\right)$, and toric morphism $X\left(\Delta^{\prime}\right) \rightarrow X(\Delta)$, where $\Delta^{\prime}$ is a smooth refinement of $\Delta$, such that the map $X\left(\Delta^{\prime}\right) \rightarrow X(\sigma)$ factors through $X(\Delta) \rightarrow X(\sigma)$. Hence, by [Kim, Lemma 2.1], $A_{\mathrm{op}}^{*}(X(\Delta)) \subset A^{*}(X(\Delta))$ and the latter is torsion-free by the previous arguments in this section.

Example 4.10. Let $\sigma$ denote the cone generated by $\{(1,0,1),(0,-1,1),(-1,0,1),(0,1,1)\}$ in $\mathbb{R}^{3}$, then $\sigma^{*}$ is actually a resolution of singularities of $\sigma$. In particular, the vertical maps are isomorphisms in Kimura's sequence. Furthermore, $E=V\left(\rho_{v}\right)=\mathbb{P}^{1} \times \mathbb{P}^{1}$, and we can explicitly compute $A^{*}\left(\mathcal{X}\left(\sigma^{*}\right)\right)$ as:

$$
A^{*}\left(\mathcal{X}\left(\sigma^{*}\right)\right)=\mathbb{Z}\left[s_{1}, \ldots, s_{4}, s\right] /\left(s_{1}-s_{3}, s_{2}-s_{4}, s_{1}+\ldots+s_{4}+s, s_{1} s_{3}, s_{2} s_{4}\right) .
$$

Hence, $s_{1}=s_{3}, s_{2}=s_{4}$, and $s=-2 s_{1}-2 s_{2} ;$ and $A^{*}\left(\mathcal{X}\left(\sigma^{*}\right)\right)=\mathbb{Z}\left[s_{1}, s_{2}\right] /\left(s_{1}^{2}, s_{2}^{2}\right)$ is clearly isomorphic to the Chow ring of $\mathbb{P}^{1} \times \mathbb{P}^{1}$ as predicted by Theorem 4.7. This calculation also directly verifies that $A_{\mathrm{op}}^{*}(X(\sigma))$ is torsion free since it injects into the ring $\mathbb{Z}\left[s_{1}, s_{2}\right] /\left(s_{1}^{2}, s_{2}^{2}\right)$ which has no $\mathbb{Z}$-torsion.

\section{Operational $K$-Theory}

In this part we prove Theorem [5.1. The proof is similar to the proof of Theorem 4.1 so we give an extended sketch. The main technical difficulty is establishing a version of the injectivity result of [ES] for operational $K$-theory

5.1. Notation and background on operational $K$-theory. We briefly recall the notation and basic results on operational $K$-theory defined by Anderson and Payne in the paper AP.

Following $\mathrm{AP}$, if $X$ is a scheme (or more generally a stack) we denote by $K_{0}(X)$ the Grothendieck group of coherent sheaves, and $K^{0}(X)$ the Grothendieck group of perfect complexes. If $X$ has an ample family of line bundles, then $K^{0}(X)$ is the same as the naive Grothendieck group of vector bundles. 
For any scheme $X$, Anderson and Payne define the operational $K$-theory op $K^{0}(X)$ of $X$ as follows. An element $c \in$ op $K^{0}(X)$ is a collection of operators $c_{f}: K_{0}\left(X^{\prime}\right) \rightarrow K_{0}\left(X^{\prime}\right)$ indexed by morphisms $X^{\prime} \stackrel{f}{\rightarrow} X$ compatible with proper pushforward, flat and lci pullback.

For any scheme $X$, there is a canonical map op $K^{0}(X) \rightarrow K_{0}(X)$ given by $c \mapsto c_{\mathrm{id}_{X}}\left(\mathcal{O}_{X}\right)$. If $X$ is smooth, then [AP, Corollary 4.5] states that this map is an isomorphism. They also prove $\left[\mathrm{AP}\right.$, Proposition 5.3] that the groups op $K^{0}(X)$ satisfy descent for Chow envelopes. In particular, the exact sequence (11) also holds for operational $K$-theory.

Theorem 5.1. For any affine toric variety $X(\sigma)$, op $K^{0}(X(\sigma))=\mathbb{Z}$.

\subsection{The injectivity theorem for operational $K$-theory.}

Lemma 5.2. If $X^{\prime} \stackrel{f}{\rightarrow} X$ is an envelope (resp. proper surjective), then the pullback map on operational $K$-theory $f^{*}$ : op $K^{0}(X) \rightarrow$ op $K^{0}(X)$ is injective (resp. rationally injective).

Proof. This follows from the formal properties of operational $K$-theory and the fact that the pushforward map $f_{*}: K_{0}\left(X^{\prime}\right) \rightarrow K_{0}(X)$ is surjective (resp. rationally surjective).

The following is the $K$-theoretic analogue of [ES, Proposition 2.10].

Proposition 5.3. If $\mathcal{X} \rightarrow X$ is a good moduli space morphism and $X$ has a resolution of singularities, then there is a pullback op $K^{0}(X) \rightarrow K_{0}(\mathcal{X}), c \mapsto c\left(\mathcal{O}_{\mathcal{X}^{\prime}}\right)$. In general this map is defined with $\mathbb{Q}$ coefficients.

Proof. Assuming $X$ admits a resolution of singularities, then there is a birational proper morphism $f: X^{\prime} \rightarrow X$ with $X^{\prime}$ a smooth scheme. Let $\mathcal{X}^{\prime}=X^{\prime} \times_{X} \mathcal{X}$ and let $f: \mathcal{X}^{\prime} \rightarrow \mathcal{X}$ be the morphism of stacks obtained by base change. If $c \in$ op $K^{0}(X)$ then since op $K^{0}\left(X^{\prime}\right)=$ $K_{0}\left(X^{\prime}\right)=K^{0}(X)$ we can identify $f^{*} c$ with an element of $K^{0}(X)$. Since pullbacks in $K$-theory of vector bundles exist for arbitrary morphisms, we obtain a class $\pi^{\prime} f^{*} c \in K^{0}\left(\mathcal{X}^{\prime}\right)$. Using the same name for its image in $K_{0}\left(\mathcal{X}^{\prime}\right)$, we define $\pi^{*} c$ to be $g_{*} \pi^{\prime} f^{*} c \in K_{0}(\mathcal{X})$. The projection formula implies that this definition is independent of the choice of resolution $X^{\prime}$.

We now obtain the $K$-theoretic analogue of the injectivity result proved in [ES].

Theorem 5.4. Let $\pi: \mathcal{X} \rightarrow X$ be a properly stable, good moduli space morphism with $\mathcal{X}$ a smooth Artin stack with generically trivial stabilizer and $X$ a scheme. Then the pullback $\pi^{*}:$ op $K^{0}(X) \rightarrow K_{0}(\mathcal{X})$ is injective after tensoring with $\mathbb{Q}$.

Proof. By [ER, Corollary 7.2], there is a commutative diagram of stacks and good moduli spaces

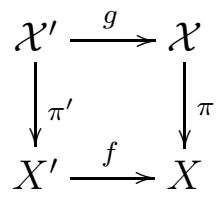

with $\mathcal{X}^{\prime}$ a smooth tame stack with smooth coarse space $X^{\prime}$ such that the map $f: X^{\prime} \rightarrow X$ is proper and birational. By Lemma 5.2, we know that $f^{*}:$ op $K^{0}(X) \rightarrow$ op $K^{0}\left(X^{\prime}\right)$ is injective after tensoring with $\mathbb{Q}$. Thus, it suffices to prove that $\pi^{\prime *}$ : op $K^{0}\left(X^{\prime}\right) \rightarrow K_{0}\left(\mathcal{X}^{\prime}\right)$ is injective after tensoring with $\mathbb{Q}$. In other words, we are reduced to the case that $\mathcal{X}$ is a smooth tame stack with smooth coarse moduli space $X$. In this case op $K^{0}(X)=K_{0}(X)=K^{0}(X)$. By the Riemann-Roch theorem for smooth schemes, the Chern character ch: $K_{0}(X)_{\mathbb{Q}} \simeq A^{*}(X)_{\mathbb{Q}}$ is an isomorphism. The Chern character commutes with the pullback $\pi^{*}: K_{0}(X) \rightarrow K_{0}(\mathcal{X})$, 
and the pullback map in Chow groups $\pi^{*}: A^{*}(X)_{\mathbb{Q}} \rightarrow A^{*}(\mathcal{X})_{\mathbb{Q}}$ is an isomorphism. Hence, the map $\pi^{*}$ is necessarily injective 1 . It follows from functoriality of the Riemann-Roch map that the composition $K_{0}(X)_{\mathbb{Q}} \rightarrow K_{0}(\mathcal{X}) \rightarrow A^{*}(\mathcal{X})_{\mathbb{Q}}$ is rationally injective.

5.3. $K$-theory of toric canonical stacks. Like the Chow ring, the $K$-theory of a canonical toric stack $\mathcal{X}(\Delta)$ admits a simple Stanley-Reisner type presentation.

The $K$-theoretic linear equivalence ideal of $\Delta$ is defined to be:

$$
K L(\Delta):=\operatorname{ker}\left(R\left(\mathbb{G}_{m}^{n}\right) \rightarrow R(G)\right) .
$$

If $e_{1}, \ldots, e_{n}$ is the canonical basis of characters, then $R\left(\mathbb{G}_{m}^{n}\right)=\mathbb{Z}\left[e_{1}, e_{1}^{-1}, \ldots, e_{n}, e_{n}^{-1}\right]$ and $K L(\Delta)$ is generated by expressions of the form $e_{1}^{a_{1}} \ldots e_{n}^{a_{n}}-1$ with $a_{1} e_{1}+\ldots+a_{n} e_{n} \in$ $\operatorname{ker}\left(X\left(\mathbb{G}_{m}^{n}\right) \rightarrow X(G)\right)$.

Definition 5.1. The $K$-theoretic Stanley-Reisner ideal of $\Delta$ is the ideal $\mathcal{K} \mathcal{Z}(\Delta)$ generated by the equivariant $K$-theoretic fundamental classes of the irreducible components of $Z(\Delta)$

If $V\left(x_{i_{1}}, \ldots, x_{i_{k}}\right) \subset \mathbb{A}^{n}$ is a linear subspace then $[V]=\prod_{j=1}^{k}\left(1-e_{i_{j}}^{-1}\right)$ in $K_{\mathbb{G}_{m}}\left(\mathbb{A}^{n}\right)=$ $\mathbb{Z}\left[e_{1}, e_{1}^{-1}, \ldots, e_{n}, e_{n}^{-1}\right]$. Using the excision sequence for equivariant $K$-theory, we obtain the analogue of Theorem 3.6.

Theorem 5.5. With notation as above,

$$
K^{0}(\mathcal{X}(\Delta)) \cong R(G) / \mathcal{K} \mathcal{Z}(\Delta) \cong Z\left[e_{1}, e_{1}^{-1}, \ldots, e_{n}, e_{n}^{-1}\right] /(K L(\Delta)+\mathcal{K} \mathcal{Z}(\Delta)) .
$$

Example 5.6. Once again, let $\sigma$ be the cone of Example 2.7, and let $\sigma^{*}$ be its star subdivision as in Example 3.9. Then $K\left(\mathcal{X}\left(\sigma^{*}\right)\right)$ is the quotient of the character algebra of $\mathbb{G}_{m}^{5}$, $\mathbb{Z}\left[e_{1}, e_{1}^{-1}, e_{2}, e_{2}^{-1}, e_{3}, e_{3}^{-1}, e_{4}, e_{4}^{-1}, e, e^{-1}\right]$, by the $K$-theoretic ideal of linear relations and the $K$-theoretic Stanley-Reisner ideal. In our case, the $K$-theoretic ideal of linear relations is generated by $\left(e_{1} e_{3}^{-1}-1, e_{2} e_{4}^{-1}-1, e_{1} e_{2} e_{3} e_{4} e-1\right)$, and the $K$-theoretic Stanley-Reisner ideal is generated by $\left(1-\left(e_{1} e_{3}\right)^{-1}, 1-\left(e_{2} e_{4}\right)^{-1}\right)$. Thus,

$$
K_{0}\left(\mathcal{X}\left(\sigma^{*}\right)\right) \simeq \mathbb{Z}\left[e_{1}, e_{2}\right] /\left(e_{2}^{2}-1, e_{1}^{2}-1\right)=K_{0}\left(\mathbb{P}^{1}\right) \otimes K_{0}\left(\mathbb{P}^{1}\right) .
$$

5.4. Completion of the proof. The proof now proceeds as the proof of Theorem 4.1. Using the analogue of Kimura's exact sequence for operational $K$-theory [AP and Proposition 5.3 , we are reduced to showing (with the same notation as in Section 4.2) that the restriction $K^{0}(\mathcal{E}) \rightarrow K^{0}(\mathcal{X})$ is an isomorphism. This fact follows from the Stanley-Reisner description of $K(\mathcal{X})$ and the same formal argument used in the proof of Theorem 4.7.

Finally, if $X$ is any smooth variety such that $A^{*}(X)$ is torsion free, then $K(X)$ is also torsion free. This follows from the fact that the natural map $A_{k}(X) \rightarrow \operatorname{Gr}_{k} K^{0}(X),[V] \mapsto$ $\left[\mathcal{O}_{V}\right]$ is surjective and becomes an isomorphism after tensoring with $\mathbb{Q}$ [Ful1, Examples 15.1.5, 15.2.16]. Hence, the same argument used in the proof of Theorem 4.9 imples that op $K^{0}(X(\Delta))$ is torsion free for any semi-proper toric variety. In particular, op $K^{0}(X(\sigma))$ is torsion free for an affine toric variety.

Example 5.7. Let $\sigma$ be the cone of Example 2.7. In Example 3.4, we showed that $\mathrm{rk} A_{*}(X(\sigma))$ $\geq 2$. Thus, by the Riemann-Roch theorem for singular schemes $\operatorname{rk} K_{0}(X(\sigma)) \geq 2$, hence op $K^{0}(X(\sigma)) \neq K_{0}(X(\sigma))$ even after tensoring with $\mathbb{Q}$.

\footnotetext{
${ }^{1}$ Note that the Chern character ch: $K_{0}(\mathcal{X}) \rightarrow A^{*}(\mathcal{X})$ is defined because the hypothesis ensures that $\mathcal{X}$ is a quotient stack by [EHKV]. The Chern character is surjective but not injective. See [Edi] for a discussion of the Riemann-Roch theorem on tame stacks.
} 


\section{REFERENCES}

[Alp] Jarod Alper, Good moduli spaces for Artin stacks, Ann. Inst. Fourier (Grenoble) 63 (2013), no. 6, 2349-2402.

[AP] Dave Anderson and Sam Payne, Operational K-theory, Doc. Math. 20 (2015), 357-399.

[CE] Thomas Coleman and Dan Edidin, Inertial Chow rings of toric stacks, Manuscripta Math. 156 (2018), no. 3-4, 341-369.

[CLS] David A. Cox, John B. Little, and Henry K. Schenck, Toric varieties, Graduate Studies in Mathematics, vol. 124, American Mathematical Society, Providence, RI, 2011.

[Edi] Dan Edidin, Riemann-Roch for Deligne-Mumford stacks, A celebration of algebraic geometry, Clay Math. Proc., vol. 18, Amer. Math. Soc., Providence, RI, 2013, pp. 241-266.

[EG] Dan Edidin and William Graham, Equivariant intersection theory, Invent. Math. 131 (1998), no. 3, 595-634.

[EHKV] Dan Edidin, Brendan Hassett, Andrew Kresch, and Angelo Vistoli, Brauer groups and quotient stacks, Amer. J. Math. 123 (2001), no. 4, 761-777.

[ER] Dan Edidin and David Rydh, Canonical reduction of stabilizers for artin stacks with good moduli spaces, arXiv: 1710.03220 .

[ES] Dan Edidin and Matthew Satriano, Towards and intersection chow cohomology on git quotients, arXiv:1707.05890, to appear in Transform. Groups (2017).

[EH] David Eisenbud and Joe Harris, 3264 and all that - a second course in algebraic geometry, Cambridge University Press, Cambridge, 2016.

[Ful1] William Fulton, Intersection theory, Springer-Verlag, Berlin, 1984.

[Ful2] _ Introduction to toric varieties, Annals of Mathematics Studies, vol. 131, Princeton University Press, Princeton, NJ, 1993, The William H. Roever Lectures in Geometry.

[FS] William Fulton and Bernd Sturmfels, Intersection theory on toric varieties, Topology 36 (1997), no. 2, 335-353.

[GS] Anton Geraschenko and Matthew Satriano, Toric stacks I: The theory of stacky fans, Trans. Amer. Math. Soc. 367 (2015), no. 2, 1033-1071.

[Gub] Joseph Gubeladze, K-theory of affine toric varieties, Homology Homotopy Appl. 1 (1999), 135-145.

[HS] Tamás Hausel and Bernd Sturmfels, Toric hyperKähler varieties, Doc. Math. 7 (2002), 495-534 (electronic).

[KP] Eric Katz and Sam Payne, Piecewise polynomials, Minkowski weights, and localization on toric varieties, Algebra Number Theory 2 (2008), no. 2, 135-155.

[Kim] Shun-ichi Kimura, Fractional intersection and bivariant theory, Comm. Algebra 20 (1992), no. 1, 285-302.

[Pay] Sam Payne, Equivariant Chow cohomology of toric varieties, Math. Res. Lett. 13 (2006), no. 1, $29-41$.

[Ric] Ryan Richey, Ph.D. thesis, University of Missouri, May 2019.

[Tot] Burt Totaro, Chow groups, Chow cohomology, and linear varieties, Forum Math. Sigma 2 (2014), e17, 25pages.

Department of Mathematics, University of Missouri, Columbia MO 65211

E-mail address: edidind@missouri.edu 